\title{
Experiencing and Interpreting: Reacting to Experiential Learning Abroad
}

\author{
Jennifer Ostojski and Carl Cilke \\ Northeastern University, Boston, MA
}

\begin{abstract}
Northeastern University (NU) in Boston, MA, offers its undergraduate students a unique summer class opportunity, entitled "Dialogues of Civilization" (DOC). DOCs are one- month or twomonth long study abroad trips, which allow students to acquire 8 to 16 credits, experiencing about a subject matter on site in an innovative hands-on experience. The University's Political Science Department offers several distinct opportunities for its budding scholars. NU's take on experiential learning differentiates itself with a holistic approach. One recent example in 2019 was a DOC to Germany to study perpetrators and remembrance in the context of the Holocaust. Students obtained an empathetic understanding through their experience. While this approach allows for a more complex set of learning objectives, DOCs provoke new pedagogical problems. Faculty together with students engage with one another continuously throughout the DOC time, boundaries between experiencing and interpreting get blurred. Students' approaches to experiencing and interpreting the environment create fundamentally different expectations. These thus developed cleavages impact the learning experience. Faculty becomes crucial in engaging with these cleavages without creating an atmosphere of exclusion. Through interviews with faculty members from the Political Science department at NU we will highlight methods of managing these experiencing and interpreting cleavages within their DOCs.
\end{abstract}

\section{Introduction}

"Do you believe that a picture is worth a thousand words? Then trust me when I say that entering the frame and becoming part of the scene is worth a thousand pictures! When all of our senses are engaged, we learn better and remember what we have learned better. We "get the full picture", and keep it too!"

(Marina Markot, Director of Northeastern's Global Experience Office)

Northeastern University in Boston, MA, is amongst the top-25 universities in the United States when it comes to sending students on study-abroad trips. The university's ranking even increases even further when looking specifically at short-term study abroad trips throughout a semester term or over the summer (IIE 2019). These rankings reflect a commitment by Northeastern University to expand and to invest more heavily in its experiential learning 
opportunities. The Northeastern 2025 agenda illustrates the university's goals of fostering and strengthening students' life-long skills and abilities to adapt and to thrive in ever-evolving globalized job market (Northeastern 2018).

Amongst many opportunities in service-learning, co-op work experiences and year-long study-abroad sessions, the university specializes in a program called Dialogues of Civilization (DOC), faculty-led short-term study-abroad sessions offered during the summer terms. DOCs date back to the early 1990s. In 1991 a Northeastern faculty leader took the first crop of students on to a short-term study abroad experience. Not much documentation exists about that early iteration of the experiential learning, but DOCs in their current form - with proper documentation - have been running for over 20 years now at Northeastern University.

Usually, these courses are approximately 5 weeks long. During that time students are enrolled in two college courses, earning, therefore, 8 credits throughout the summer in this highimpact learning environment. Courses are either an adaptation of already existing courses on campus during the regular spring and fall semesters, or they are newly created based on the research interests of the faculty. In the upcoming Summer 2020 term, Northeastern University will run 70 DOCs in over 30 countries around the world. All major fields are represented (from engineering, over humanities, to design), yet the majority of the classes offered are in the social sciences and humanities.

Institutionally, the Global Experience Office (GEO) of Northeastern supports faculty in the creation and execution of the trip. This includes the preparation of a trip proposal, budgets, itineraries, health and safety concerns but also in the aftermath of the trip an evaluation of student and faculty surveys about the trip. The office does mandate certain aspects in the 
structuring of the study-abroad trip: cultural activities and site visits have to be present on the schedule, as GEO in accordance with experiential learning wants students to immerse themselves in a forgein culture, rather than simply having traditional classroom activities in a different country; the office demands pre-departure meetings so students and faculty can interact and bond to enhance social cohesion on the trip; lastly, GEO strongly encourages and provides resources for faculty to schedule debrief sessions on the trip for the students to reflect.

On the whole, though, GEO refrains from directly interfering or advising on syllabi or pedagogical questions. Unless an actual emergency situation arises, the moment students and faculty step on the plane, is it up to the faculty to construct and provide a meaningful trip in which the presented experiences lead to positive learning outcomes. The faculty becomes the sole bearer of learning principles and norms, and it is up to them and their intentions that make or break a trip.

We demonstrate through our research that the choices a faculty makes demarcate the limitations and possibilities of experiences had and reflections made on a trip. We show that experiences can vary across a spectrum as well as reflections can be conducted in varying ways. Our findings show the DOC program overall is unique in its existence, and yet, at the same time, the choices made by each faculty member to create a unique pedagogical venture falls in line with the mainstream literature on the importance of the faculty. They are not just a tour guide and facilitator of material. Rather, they are the orchestra and the conductor at the same time.

\section{Literature Review}

The concept of experiential learning has been finding ground in the pedagogy literature for over a century now. It was Dewey’s work "Experience and Education" (1938) that created 
the foundation for understanding how valuable experiential learning is for students in the knowledge creation process. The scholar rejected the notion of the traditional classroom setting, in which information is passively transmitted from the educator to the student. Knowledge creation in a student could not and would not occur through this manner. In order to facilitate the creation of knowledge a student would have to feel included and in control when participating in a lesson. Dewey advocated for an experiential learning environment, in which the student was exposed to experiences that would encourage the creation of knowledge in an active manner. The learning environment, therefore, paired with the practices created by the instructor were not just a meaningless exercise but at the core of the learning process (Dewey 1938, Roberts 2003 ).

Dewey stressed rightfully that a faculty's impact on a learning environment will not always have positive outcomes. Sometimes, choices made by a faculty can cause undesired outcomes which do not allow the students to meaningfully create knowledge. Sometimes a poorly designed experience can guide students off the tracks and worsen their learning outcome. Nonetheless, the learning process - be it good or terrible - relies heavily on the preparation and the intentions of the educator. Their mark alone on the learning process can advance or inhibit the learning cycle through experiential learning. Experience, therefore, does not necessarily equal experience. Rather, an experience had to be constructed in a way that allows a student to not just take in the necessary class topic but to grow personally and create the ability to form knowledge that will be useful in future applications (academic as well as professional) (Dewey 1938).

Key, though, in his critique about the traditional classroom was not just its passively designed learning environment. In order to actively - experientially - teach a student body, so Dewey (1938), educators had to change the insular environment that a traditional classroom 
provides. Simply sticking to the four walls of the traditional classroom and limiting oneself to abstract examples in a textbook would not allow for actual learning to occur. The theoretical trend and the lack of real-world examples deprives a student of a connection which later on would allow them to practice their application skills in the learning cycle (Dewey 1938). There are many different ways in which experiential learning can be facilitated.

Wurdinger and Carlson (2009) have outlined what approaches to experiential learning can look like in theory, categorizing them as such: active learning, problem-based learning, project-based learning, service learning, and place-based learning. In praxis, especially in the context of higher education, these approaches can be transformed into specific classroom activities created by an educator, which actively involve the students and allow them to take on an active role in a specific class segment. These can include presentations, group activities, guest speakers and panels. They can also involve the creation of individual research projects, allowing the students to gain actual ownership of their knowledge creation. Additionally, experiential learning can be facilitated through the promotion of internships or the implementation of visits to practical sites of learning (i.e. laboratories, museums, etc.). An emerging field in experiential learning is also the incorporation of service-learning initiatives, in which students are engaging with non-profit organizations within their communities (Georgia State University 2019).

Lastly, though, and most importantly in the context of Dewey's critique of insular learning environments, is the rising trend for United States college students to embark on longterm and short-term study abroad trips to immerse themselves in new cultures and new environments (Georgia State University 2019, IIE 2019). Long-term and short-term study abroad trips immensely impact a student's learning outcome for the better. "A study abroad program yields a variety of such educational outcomes, including stimulating curiosity and critical 
thinking, gaining insights into a new cultural perspective, and putting classroom knowledge into real world practice" (Shostya, Morreale 2017). They provide an environment of 'high impact' learning, which allow a student to experience personal growth in their cultural awareness, their critical thinking, and their sense of self (Kuh 2008).

Experiential learning, in which learning is defined as "the process whereby knowledge is created through the transformation of experience. Knowledge results from a combination of grasping and transforming experience" (Kolb 1984), has become an aspirational norm across higher education campuses in the United States and around the world. Kolb \& Kolb (2005) provide a cyclical model for experiential learning. A student will have an experience (1), then they reflect on that experience (2), they will conceptualize the experience, meaning what they can take away from it (3), and lastly they will attempt to apply the concept in a new setting or a new experience (4) - thus perpetuating the cycle. With each iteration of the cycle, the learning becomes more complicated as experiences and reflections build on one another (Kolb, Kolb 2005, Georgia State University 2019)

Faculty-led short-term study abroad trips (FLSTSAT) are a type of experiential learning which illustrates the complexity of experiential learning and its effectiveness in the creation of knowledge for students. Usually they last eight weeks or less and occur either during the semester or during a University's summer term. Since the 2005/2006 academic year, short-term study abroad trip numbers have increased amongst American higher education institutions. The two most popular options are short-term trips abroad within the academic year and short-term trips abroad during a university's summer term (IIE 2019). Echoing Dewey's critique of misguided experiences and unintended consequences in the reflections provoked, Augilar and Gingerich (2002) argue that "while study abroad usually involves some form of active learning, 
this does not always lead to experiential learning as some programs do not allow students critical time for interaction and reflection." The necessary steps and critical junctures, as outlined by Kolb \& Kolb (2005) can potentially not be fulfilled and met in a shortened stay: experiencing and reflecting may not occur or not in the way an educator has set out the experience to be taken in (Augilar, Gingerich 2002).

While a long-term study-abroad trip, usually conducted by themselves or in a smaller groups, forces a student to fully immerse themselves in the environment they travelled to simply due to the nature of these long-term trips -, an FLSTSAT may prevent a student to have to fully immerse and experience the culture as intended due to short amount of time an FLSTSAT provides. The high impact nature of an FLSTSAT, meaning constantly having new experiences in a short amount of time and in a completely new environment, can contribute to the lack of reflecting on the student's side and, thus, ultimately the learning process itself (Augilar, Gingerich 2002, Shostya, Morreale 2017).

There are other factors to be considered as potential obstacles in the knowledge creation process on short-term study abroad trips which speak to the general problematique of short-term study abroad trips. For one, embarking on short-term trips does not permit enough time to appropriately encounter a local culture. Since Universities are investing and expanding their offerings to respond to the need of preparing students for an ever evolving globalized world, the short-sighted travel opportunity can perpetuate "hierarchies of power and colonialism" (Pipitone 2018) and narrate an experience that is closer to tourism rather than scholarship, while simultaneously ignoring the nuances of historical socio-economic variables. Other factors include the "glorification of immersion" (Pipitone 2018), pursuing an artificial form of immersion, or insisting on the importance of the student's personal growth (Pipitone 2018), 
which can be in itself a difficult balance to strike as a study-abroad trip at its core is about a student's ability to adapt and grow to a new world (Pipitone 2018).

Less explored but still manifested issues in regard to factors impacting the learning experience on short-term study-abroad trips are aspects, such as, for instance, the lack of general travel experience by American students outside the United States, resulting in a lack of cultural sensitivity. Numbers are still relatively low when it comes to the general United States undergraduate population. While they dipped considerably in 2008 due to the economic crisis, they have been slowly increasing in the past 5 academic years (Musto, Gundy 2018, IIE 2019). Further, a short-term study abroad trip, will eventually encounter the obstacles of cliques forming or other social-group dynamics developing, which can impact the learning environment. Additionally, the increase of technological distractions and the constant ability to stay connected can even the technological distractions that accompany students on these trips which inhibit the immersion experience of a student in their study abroad environment. Additionally, preconceived notions of the students about the places visited on the trip, either formed on their own or through the influences of parental/familial figure heads, can impact the way a student approaches the learning experiences abroad.

Some of these aspects are out of the educator's hands. A large portion, though, is up to the educator to create a cohesive and holistic environment, which acknowledges the aspects of short-term cultural immersion, the respect a local culture deserves, or any potential toxic group environments that can impact the abroad learning environment. This conclusion is largely acknowledged and agreed upon in the literature (see, for instance, Anderson, Lorenz, White 2016). The educator's choices in experiential learning not only set and create the learning environment itself, but they remove as best as possible obstacles that can impact the learning 
itself. This includes the obstacles created by the (short) length of study abroad trips (Gibson, Strange 2017); the group dynamics amongst the students as well as the relationship with the professor (Ritz 2011, Abualrub et. al. 2013, Shostya, Morreale (2017); or the cultural implications related to a study abroad trip (Anderson, White 2016).

These types of research, though, address factors which make up the learning environment from the outside, without necessarily looking directly at the learning process, as outlined above. Crucial to the experiential learning process are the first two steps: experiencing and interpreting (reflecting). Educators are not just tasked with creating a 'safe environment' surrounding the students as they experience and interpret; they are required to meaningfully structure experiences and spaces to interpret. Without properly paying attention to these steps, conceptualizing and application will either be lacking meaning or simply not occur. The study abroad trip then serves no educational purpose that advances learning objectives.

\section{Methodology}

Using Kolb’s (1984) spatial differentiation of experiencing and learning (as well as conceptualizing and application), we advance the field of research through our qualitative research, in which we interview faculty leaders on short term study abroad trips, Dialogues of Civilization, as offered by Northeastern University in Boston, MA. We discuss with our interviewees their conception of experiencing and interpreting, how their understandings of these concepts guide their pedagogical choices on these trips, and challenges they encounter on shortterm study abroad trips in terms of the experiential learning process. We analyze their responses and attitudes, and, subsequently, we provide practices and prescription which faculty leaders can incorporate in the planning of their own short term study abroad trips. In our research paper we 
have anonymized our interviewee's identity and other indicators, especially in regard to their study abroad topics, that may hint at their identity.

\section{Experiencing and Interpreting}

\section{1) Structuring Experiencing and Interpreting}

Despite a relatively small group of faculty interviewees, one of our most important findings in our research is that every single faculty member structure their DOC differently. Their varying conceptions and creations of experiential learning have resulted into two distinct structuring models for short-term study abroad trips. We can broadly separate those into the two following: on one hand the "mobile classroom model" (MCM), and on the other hand the "home base model" (HBM). Choosing either one has both positive outcomes and negative shortcomings.

In regard to the HBM, one interviewee stated, "I like having the home base because it creates you know, you create accountability, you create a learning environment. [the students are] used to being on campus, it's nice that we have the benefit of a campus to be there at."(Interview 8). The home base model typically involves a dominant university partner in the foreign country visited. These types of partnerships with foreign universities can have varying levels of formality. Some DOCs are designed to be integrated into that University's summer curriculum, meaning students take local classes facilitated by local university professors. Other times, the partnership means simply that Northeastern University rents out dorms and classroom space, with the Northeastern faculty leader hosting all lectures (Interview 1, 22).

The mobile classroom model is quite different in its approach. The faculty leaders

chooses to minimize the classroom as much as possible. One interviewee stated, "just the whole 
idea of spending, you know, hours a day into classroom. So, if we're in a classroom for an hour or two over the day that that, for me is like a pretty long time." (Interview 15). The design of an MCM is logistically completely different from an HBM. Classroom 'lectures', for instance, take places in museums, in government offices, in conversation with practitioners, and at sites where events have actually occurred. The MCM keeps students and faculty more on the move and in the field. Often times, these dialogues do not stay for their entirety in one city, but rather, they hop around the country or even multiple countries to gather as much exposure as possible.

The choice between the HBM and the MCM reflects the faculties' varying pedagogical understandings on how experiential learning should be structured on FLSTSATs. These two models are our two broad frameworks, yet even within those, plenty of variation is visible. Despite a set of external factors (i.e. institutionally as discussed above), each interviewee has clearly expressed that there is agency for them to personalize their DOCs. This means that no two structures are the same, meaning that no two DOCs are the same. The ability to customize and design their own experiential learning structures and their conceptualizing of that important learning concept, allows then a visible variation between how faculty regard and implement the interpreting pillar into their study-abroad trips.

\section{2) Understanding Experiencing}

Every one of our interviewees has a different understanding of experiencing as an essential part of the experiential learning cycle. The faculty demonstrate their different understanding in both how they communicate about experiential learning, and how they design their trips. We structure these variations on the spectrum of full immersion to full facilitation. This spectrum does not align with either of the two structural typologies (HBM or MCM). 
Rather, we observe that within these two structural models all types of experiential learning have been incorporated by the DOC faculty leaders.

One interviewee defines full immersion as followed: "I call it immersion, ... It's really like taking them out of the classroom to the actual subtleties of life and teach them to use their own lens critically to look at different realities at different parts of the world."(Interview 10). This understanding of immersion is that the faculty's understanding of experiential learning is really to dive into that experience as fully as possible. As one instructor argued, "I have a principle, when we are spending only a month in a totally different socio political context, climate [and] geography. The less time they spend indoors, especially on their own in their hotel rooms, reading and writing, the better."(Interview 18).

One facet of this understanding is that it is difficult to have any objective measurement in a student's experience. An interviewee expressed that,

"the way I look at the Dialogue and study abroad in experiential education means that... My task is not to elicit a particular experience. Nothing in my program ....is set out in a way that I seek everyone to fall into a particular experience. I look at my program as wanting to [sic] create an environment within which any experience is possible, and as a collective, we then travel through it, you know, as sort of in alignment."( Interview 6)

When an instructor understands experiencing as immersion, then the ability to create the opportunity for immersion becomes the goal of the experience. Rather than trying to illicit a certain understanding, or a unified and collective experience, the experiences on the trip become extremely personal to the individual student. So, particular site visits or 'activities' are specifically designed for this individualistic experience. One example is the goals set out by one interviewee focused on immersion, " they could just be there, which is already good, but still, I'm trying to hit as many boxes to be sure, they should be able to walk around to touch, they should 
be able to go and look at the documents. They should be able to speak; they should be able to be confronted with a particular emotional experience."(Interview 6). Rather than a guided or facilitated experience, the purpose is to allow for every student the opportunity to experience it for themselves to the degree they chose to experience it.

In the middle of our experience spectrum a is another approach, namely a more structured approach to immersion. The major difference for this approach is that the immersion itself is designed to include more than simply experiential learning. In this approach, the experiences are designed to illicit a specific reaction or connect. One instructor described it as, "we try to combine experiential learning and the place seeing what's happening."(Interview 23). This style includes the elements from the immersive approach, but every experience is designed to bring greater clarity to the specific academic concepts. The students can be evaluated on how much they have gleamed from that specific activity, rather than the goal being deeply personal.

Finally, on the other side of our spectrum, meaning the facilitating sector, instructors have understood experiencing in a much more structured or controlled way. One instructor designed their approach as "this program is kind of taking that to heart that we want this to be an experiential process, but we don't just want to dive in for five weeks and not ever come up for air." (Interview 9)

There is an understanding that the best way to facilitate experiencing is not to simply dive into to a new environment. Faculty actively resists full immersion. The strategy here used is to create a sense of routine for the students, which includes constantly cycling back and forth from the classroom to the experiences, and then back to the classroom. This means in practical 
terms that the students' morning contains several hours of traditional classroom time, and in the afternoon they travel to an experience (Interview 12,17).

This routine allows for those experiences to be more easily incorporated into the much familiar course structure. An approach like that results also in specific types of experiences, as these include more familiar (and more comfortable) site visits, guest lectures and presentations from relevant to the topic actors(Interview 8,12). This approach also allows to hit specific academic goals more neatly, as these types of experiences are easier to include into a student's learning cycle due to their obvious nature.

A key observation made when analyzing the faculties' choices on the experience spectrum is how they view and discuss a student's workload and potential overload. On the facilitating end we see opinions about student work, such as, "the idea is they're kind of getting a little bit of time to really dive in really have this experiential and reflection process, but not necessarily get overloaded by it." (Interview 1). And also "My role [as faculty leader] first is to keep you safe. Okay, then healthy, and then to have a positive learning environment." (Interview 22). These instructors believe that experiencing can be best facilitating by being very conscious of overload. They, therefore, structure the classroom and experience sections carefully and with the focus on maintaining a feasible workload and a not-overpowering daily schedule. This includes, for instance, to end a day's work either at $4 \mathrm{pm}$ or $5 \mathrm{pm}$ after some lecture time and some site experiences. Faculty choses to do so to ensure that students to not become physically and mentally exhausted. The acknowledge that within the cycle of experiential learning 'too much experience' can cause burnout and those counteract the whole purpose of experiential learning. 
Faculty on the immersive end of the experience spectrum view the activities portion of their trips rather differently. One instructor described their workload as, "once you get in the field, once you once you're there with the students, and you're immersed. Because our days are pretty intense, and we will get up, you know, leave the hotel at eight won't be back till 8pm. So, 12 hours pretty much on the road most days." (Interview 19). Instead of being concerned with physical or mental exhaustion, faculty believes that full immersion and hefty work/experience loads are key to experiential learning. Free time for students is kept to minimum. Instead, frequent location changes and activities are key to immersing fully into the foreign environment.

\section{3) Understanding Interpreting}

A faculty's position on our experience spectrum (immersion to facilitating) connects directly to their understanding of interpreting. The purpose of interpreting is defined in this context as the reflection on experiences, which is necessary to eventually be able to conceptualize and apply learned concepts to new experiences. Different experiencing approaches on DOCs create varying types of constraints on the ability to interpret. The faculty, nonetheless, can counter act some limitations on reflection through specific structural choices. Once again, we observe different attitudes in the importance of reflecting and the way students should encounter this process.

A key difference between faculties' choices is how they see their position in the reflection itself: either as a by them guided process or as student-led deeply personal process. GEO suggests that faculty incorporate reflections in some capacity, but the how is left to the instructor themselves. As one instructor emphasized the importance of creating spaces for reflection, "debriefing after the event, I want to create some things again, using sort of using slack, using shared communication, post some questions to have them pre- and post-process 
things, doing some low stakes reflection... and thinking about it as one way that I can at least give them the tools to do that."(Interview 9). So, after every experience, there is a designed and structured part of the course where students are primed to be reflecting on their experiences.

This approach, though, is time intensive and to avoid it, different faculty will choose to reflect and to debrief occasionally (not in a structured or scheduled manner) or after the most intense experiences. One interviewee described this debrief after one of their powerful experiences, "Right, then we'll have a debrief. Okay. So, what do we see today? How did we read last night? You know, what you know, [sic], what do you notice? procedures? So, having a debrief, but it's based on then combining, they've read about it right on the night before we go there, plus the experience of being there on the site."(Interview 15).

By using the active debrief somewhat occasionally - sometimes in the classroom, sometimes literally in a field -, faculty are reinforcing potentially in certain instances particular experiences as being more impactful than others. The emphasis on reflection is less so than the importance of the experience itself. There seems to be an assumption that reflection and interpreting will simply happen along the way for each student individually.

Alternatively, the decision to debrief after almost all events is an understanding of interpreting that aligns with the facilitating understanding of experiencing. Students are not simply left to their own devices in the interpreting phase of learning. Rather, faculty ensures that certain key interpretations are either achieved individually or collectively, as to confirm that the group moves together through the trip and a lowest common denominator of learning is occurring unilaterally throughout the trip.

Instructors, who understand experiencing as immersion, seem to rely on external tools to help solidify the interpreting portion. This is mainly done through scheduled assignments and an 
assigned final project for the students. Final projects are a way for students to demonstrate for one their different interests and passions but also how they have academically reflected on the concepts learned and how they chose to apply them to their own research. It allows for faculty to check in at the end of a trip to see how much learning has actually occurred.

Some faculty chose their assignment to be specifically reflective papers throughout the DOC (Interview 4,7). These papers are not designed to test the academic course requirements (factual knowledge), but rather, they are assigned to create the reflection necessary for the interpreting part of the learning cycle. One instructor described their approach, "I do writing, [it] is writing intensive because the whole point of this, of a dialogue, is to experience it and reflect upon it and process it. Make sense of it. Raise Questions about it, draw some conclusions about it. But also draw conclusions about yourself.“ (Interview 11). Those reflections elicited through these types of papers are deeply personal, as they do neither have a particular instructed angle nor is there a requirement to discuss something in particular. The process of reflecting becomes, therefore, more meaningful than the actual product created. The students should be immersed in these new experiences and drawing deeply personal conclusions, rather than necessarily connecting those concepts back to the academic components.

\section{Discussion}

Northeastern University stands out in the way it structures and advertises its FLSTSATs. The notion of picking up a Northeastern classroom and its faculty and transporting the student body into a foreign environment to learn on site is rather unique in the broad landscape of shortterm study abroad trips. The positive outcomes of just generally embarking on an international trip to learn hands-on what a specific topic means in a g(l)ocal context are undisputed. And yet, 
our interviewees illustrate that experiential learning is a vastly more complex process than presumed.

The necessary components of the learning cycle, experiencing and interpreting, are impacted in their depth and width by the faculty's choice to choose either the HBM or the MCM. The choice for a particular model is impacted by two key components: one, the faculty's key intentions in what kind of experiences and reflections they aim for, and two, a set of additional variables that contribute to the choice of the faculty as well. Our interviews demonstrate that the faculty's personality, their research interests, their ideas for a student body, as well as the topic taught on site contribute tremendously to the faculty's ultimate decision to pick either the HBM or MCM. This choice defines their primary and path-defining pedagogical choice which will impact the cycle of structuring experiencing and learning.

The cycle is divided into two halves. Initially, the faculty's intent impacts the choice for a model and thus defines the limitations of experiences and reflections through their chosen model. Almost tautologically, the demarcation then justifies the chose model, which in turn constitutes the other half of the experiential cycle. The chosen model (either HBM or MCM) is additionally impacted by outside forces, such as institutional constraints (i.e. GEO), financial issues, and logistical obstacles, which in turn create a potentially different reality of constrained experiences and reflections on the student's side of being on the trip. Their part of the cycle is where the actual learning occurs. 
All of our interviewees have either run the DOCs multiple times or aim to run them frequently. Therefore, the student's reality of experiencing and interpreting has a lasting impact on the instructor's intent of choosing a model for the next FLSTSAT. Their realty, therefore, either reinforces a chosen model or impacts the faculty to alter certain aspects of the trip. Either way, the more times a specific trip has occurred to more it becomes institutionalized in its models and its limitations of experiencing and reflecting. The model below illustrates what occurs on Northeastern University's DOCs.

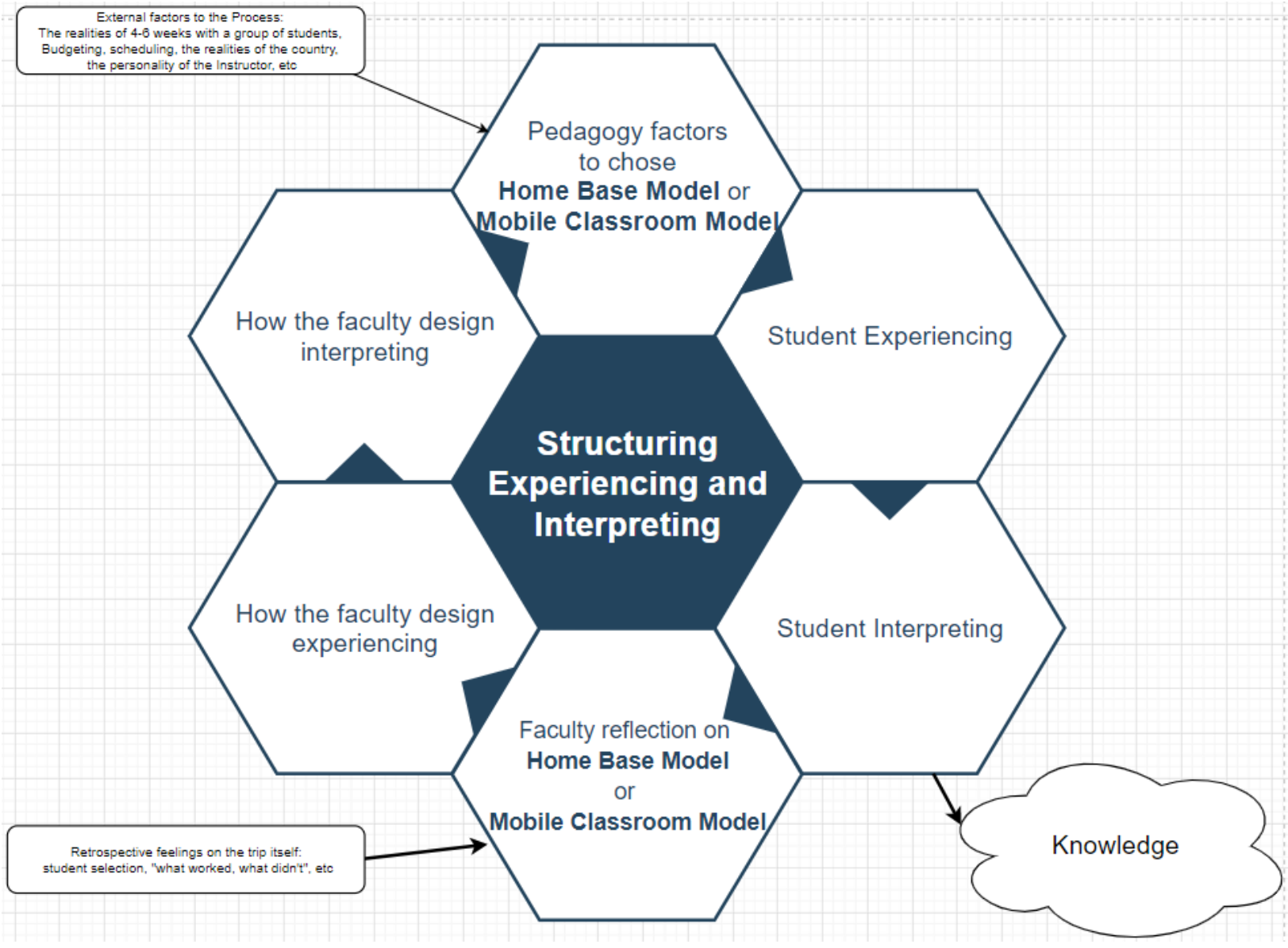

Model 1: Structuring Experiencing and Interpreting 
This model demonstrates not just a uniqueness to the way the University has introduced a successful and improving short-term study abroad scheme. It also shows that each individual trip is not just unique in terms of the destination and topic taught but in its structuring of experiences and interpreting. Thus, the learning and knowledge acquisition process is completely different for each student group across the board.

Our interviewees showed preferences in the way they let students come onto the trip, meaning they weigh the components of the institutionally mandated process differently. Generally, each undergraduate student has to apply to a DOC with an essay and, later, they will undergo an interview. The faculty chooses how to measures these components. Faculty choosing to facilitate experiences are specific in their student selection. They emphasize the importance of a student's major, their research interests, and their ability to work and travel well in groups. Faculty, who value immersion experiences, choose a more inclusive route of student selection, arguing, "it's difficult to deny students to the world of experiential education because the very fabric of that is that you want to explore the world and like who are you to say, "No, not you?"'( Interview 6)

This particular decision-making process can be correlated with the immersive side of the experiencing spectrum, meaning that if the experiencing is understood as a deeply personal process, then it is impossible to know who will be most receptive to material. Faculty with this intention in mind believed that little can be gleamed from an undergraduate in the standard interview and essay portion of the DOC application. One instructor even believes that from their previous experience that the student selection process is just completely random and that it does not help at all. The student body make-up and their cohesion throughout the trip is simply a draw of luck (Interview 4,21). 
Another variable in the structure of the trip is the topic of the trip itself. Northeastern's DOCs are not simply language trips or destination trips to a specific country. The topic itself dictates the location chosen by faculty. Student, therefore, self-select partially into the location but more importantly into the topic at hand. The relationship of the faculty with the topic contributes as well to the structuring of their experiential learning and their emphasis on reflections. As one interviewee stated,

“... so I'm teaching this topic and I am very interested in everything that you set up, right? To think about what is the benefit of being in these places and in my topic, in particular, in a society that is already the foundation that argues actually you must go and see it. You should not read a book about it; you need to go and see it for yourself."(Interview 6)

Another interviewee reflected on the choices made on the trip in regard to their experience and their intentions to show the students a specific niche in the field studied, stating that while students are exposed to other material, the course was largely designed to reflect the instructor's angle and their research interests, "I'm kind of selfish, you know, it's my DOC." (Interview 23). The faculty's personal relationship to the topic, therefore, cannot be ignored in the structuring of the trip.

\section{Conclusion}

Our conceptions of experiencing and interpreting come from relatively similar sources of data. It involves instructors from the Political Science department, all operating FLSTSATs for the same amount of time, utilizing the same university resources. Yet, the conceptions of how to run a DOC are very different. All these differences are connected to that instructor's individual pedagogical preferences. Which leads to the question, how much do those differences matter? The nature of our research design prevented us to speak to any former students or obtain any evaluation data. That is one limitation of our paper, as we were unable to connect the faculty's 
differences to any objective measurement of success. In fact, all our instructors are conceiving of success very differently. Some see it as a successful research project, where the students have incorporated field work data. Whereas others view it as a deeply reflective assignment, in which the transformative nature of the trip is ultimate goal.

But these variations are quite large, even among a small group of faculty members, in merely one department. What is interesting about this variation is that it is directly connected to that individual instructor. When these faculty members are discussing how they create their DOC, they will describe their choices as having their pedagogical understanding of experiencing and interpreting as driving their course creation. But we not quite sure how true that is. We believe that before the pedagogical interpretation begins, instructors have already decided on their classroom model, either the Home Base Model or Mobile Classroom Model. The choices that they are making, when planning their DOC, are then reinforcing their own decisions. Our model is path dependent. Once faculty have chosen a structure, they are reinforcing and adjusting their choices annually - still within one classroom structure.

We are not making an evaluation on which structure is better for the experiential learning cycle. These DOCs are in different countries, studying different topics, and should not be cookie cutter replications of each other. But, the decision of choosing a model, leads the students to have very different experiences. The fact that they are different is something that should be further incorporated into the process. The resources provided to faculty treat every DOC similarly, even though they are in fact pedagogically diverse. GEO's program managers appear to be assigned only by country or region, rather than by pedagogical methods chosen. Resources for students when they are abroad are relatively identical. Institutional guidance for faculty acts as though they are the same thing. We believe that FLSTSATs should have these pedagogical 
distinctions incorporated directly into the construction and support for these programs to not just support the faculty more appropriately but also provide the students with clearer information about their experiential learning experience.

From what we have researched in our own case study at Northeastern University, the DOC institutional structure might be unique to the University itself. However, the pedagogical obstacles in structuring an FLSTSAT are in our opinion generalizable to other institutions which engage in similar experiential learning opportunities. 


\section{Bibliography}

Abualrub, Iyad, Berit Karseth and Bjørn Stensaker. "The various understandings of learning environment in higher education and its quality implications." Quality in Higher Education, vol. 19, no. 1, 2013, pp. 90-110.

Anderson, C., Lorenz, K., \& White, M. "Instructor Influence on Student Intercultural Gains and Learning during Instructor-Led, Short-Term Study Abroad." Frontiers: The Interdisciplinary Journal of Study Abroad, vol. 28, 2016, pp. 1-23.

Aguilar, A. L., and Orval Gingerich. "Experiential pedagogy for study abroad: Educating for global citizenship.” Frontiers: The Interdisciplinary Journal of Study Abroad, vol. 8, 2002, pp. 41-82.

Dewey, John. Experience and Education. Indianapolis, IN: Kappa Delta Pi, 1938.

Georgia State University. 2019. "Types of Experiential Learning.” Georgia State University. https://myexperience.gsu.edu/faculty/resources/types/. Accessed 30 Jan. 2020.

IIE. 2019. "Leading Institutions." The Power of International Education. https://www.iie.org/Research-and-Insights/Open-Doors/Data/US-Study-Abroad/LeadingInstitutions. Accessed 30 Jan. 2020.

IIE (1). 2019. "Duration of Study Abroad.” The Power of International Education. https://www.iie.org/Research-and-Insights/Open-Doors/Data/US-StudyAbroad/Duration-of-Study-Abroad. Accessed 30 Jan. 2020.

Kolb, David A. Experiential Learning: Experience as the Source of Learning and Development. . Upper Saddle River, NJ: Prentice Hall.

Kolb, Alice Y., and David A. Kolb. "Learning Styles and Learning Spaces: Enhancing Experiential Learning in Higher Education." Academy of Management Learning \& . Education, vol. 4, no. 2, 2005, pp. 193-212. JSTOR, www.jstor.org/stable/40214287. Accessed 30 Jan. 2020.

Musto, Pete, and Dorothy Gundy. 2018. "Number of US Students Studying Abroad Continues to Grow." VOA Learning English. https://learningenglish.voanews.com/p/5373.html. Accessed 30 Jan. 2020.

Northeastern University. 2020. "Northeastern 2025." Northeastern University. https://www.northeastern.edu/academic-plan/. Accessed, 30 Jan. 2020, 
Ritz, Aixa A. "The educational value of short-term study abroad programs as course components." Journal of Teaching in Travel \& Tourism, vol. 11, no. 2, 2011, pp. 164178.

Shostya, Anna, and Joseph C. Morreale. "Fostering Undergraduate Research Through a FacultyLed Study Abroad Experience." International Journal of Teaching and Learning in Higher Education, vol. 29, no. 2, 2017, pp 300-308.

Strange, Hannah, and Heather J. Gibson. "An investigation of experiential and transformative learning in study abroad programs." Frontiers: The Interdisciplinary Journal of Study Abroad, vol. 29, no 1, 2017, pp. 85-100.

Wurdinger, Scott D, and Julie A. Carlson. Teaching for Experiential Learning: Five Approaches That Work. Lanham, Maryland: R \& L Education, 2009. 